\title{
Logical and Methodological Issues Affecting Genetic Studies of Humans Reported in Top Neuroscience Journals
}

\author{
Clara R. Grabitz ${ }^{1}$, Katherine S. Button ${ }^{2}$, Marcus R. Munafò ${ }^{3}$, Dianne F. Newbury ${ }^{4}$, \\ Cyril R. Pernet ${ }^{5}$, Paul A. Thompson ${ }^{6}$, and Dorothy V. M. Bishop ${ }^{6}$
}

\begin{abstract}
Genetics and neuroscience are two areas of science that pose particular methodological problems because they involve detecting weak signals (i.e., small effects) in noisy data. In recent years, increasing numbers of studies have attempted to bridge these disciplines by looking for genetic factors associated with individual differences in behavior, cognition, and brain structure or function. However, different methodological approaches to guarding against false positives have evolved in the two disciplines. To explore methodological issues affecting neurogenetic studies, we conducted an in-depth analysis of 30 consecutive articles in 12 top neuroscience journals that reported on genetic associations in nonclinical human samples. It was often difficult to estimate effect sizes in neuroimaging paradigms. Where effect sizes could be calculated, the studies reporting the largest effect sizes tended to have two features: (i) they had the smallest samples and were generally underpowered to detect genetic effects, and (ii) they did not fully correct for multiple comparisons. Furthermore, only a minority of studies used statistical methods for multiple comparisons that took into account correlations between phenotypes or genotypes, and only nine studies included a replication sample or explicitly set out to replicate a prior find-
\end{abstract}

\section{INTRODUCTION}

Studies reporting associations in humans between common genetic variants and brain structure or function are burgeoning (Bigos, Hariri, \& Weinberger, 2016). One reason is the desire to find "endophenotypes" that provide an intermediate step between genetic variants and behavior (Flint \& Munafò, 2007); to this end, it is often assumed that brain-based measures will give stronger associations than observed behavior because they are closer to the gene function. Furthermore, it is now cheaper and easier than ever before to genotype individuals, with many commercial laboratories offering this service, so neuroscientists interested in pursuing genetic

\footnotetext{
${ }^{1}$ Radboud University Nijmegen, ${ }^{2}$ University of Bath, ${ }^{3}$ University of Bristol, ${ }^{4}$ Oxford Brookes University, ${ }^{5}$ The University of Edinburgh, ${ }^{6}$ University of Oxford
}

ing. Finally, presentation of methodological information was not standardized and was often distributed across Methods sections and Supplementary Material, making it challenging to assemble basic information from many studies. Space limits imposed by journals could mean that highly complex statistical methods were described in only a superficial fashion. In summary, methods that have become standard in the genetics literature-stringent statistical standards, use of large samples, and replication of findings-are not always adopted when behavioral, cognitive, or neuroimaging phenotypes are used, leading to an increased risk of false-positive findings. Studies need to correct not just for the number of phenotypes collected but also for the number of genotypes examined, genetic models tested, and subsamples investigated. The field would benefit from more widespread use of methods that take into account correlations between the factors corrected for, such as spectral decomposition, or permutation approaches. Replication should become standard practice; this, together with the need for larger sample sizes, will entail greater emphasis on collaboration between research groups. We conclude with some specific suggestions for standardized reporting in this area.

studies need not have their own laboratory facilities to do this. The ease of undertaking genetic association studies is, however, offset by methodological problems that arise from the size and complexity of genetic data. As Poldrack et al. (2017, p. 115) cautioned with regard to neuroimaging data, "the high dimensionality of fMRI data, the relatively low power of most fMRI studies and the great amount of flexibility in data analysis contribute to a potentially high degree of false-positive findings." When genetic approaches are combined with neuroscience methods, these problems are multiplied. Two issues are of particular concern.

The first issue is that the field of neuroscience is characterized by low statistical power (Button et al., 2013), where sample sizes are often too small to reliably detect effects of interest. Underpowered studies are likely to miss true effects, and where "significant" effects are found, they are more likely to be false positives. Where 
common variants are associated with behavioral phenotypes, effect sizes are typically very small; robust associations identified in genome-wide association studies (GWAS) typically account for less than $0.1 \%$ of phenotypic variance (Flint \& Munafò, 2013). These reach genome-wide significance only when very large samples are used with this method. If we have a single-nucleotide polymorphism (SNP) where a genetic variant accounts for $0.1 \%$ of variance (i.e., $r^{2}=.001$ ) and we want to reliably detect an association of that magnitude, simple power calculations (Champely, 2016) show that we would need a total sample of 780 cases to detect the effect with $80 \%$ power at the .05 level of significance. If we had 200 participants (100 for each of two genotypes), then our power to detect this effect would be only $29 \%$. Although it is often argued that effect sizes for neuroimaging phenotypes may be larger than for behavioral measures, a recent analysis by Poldrack et al. (2017) suggests caution. They found that, for a motor task that gives relatively large and reliable activation changes in the precentral gyrus, $75 \%$ of the voxels in that region showed a standardized effect size (Cohen's $d$ ) of less than one, and the median effect size was around .7; for other well-established cognitive tasks, the median effect sizes for a specified ROI ranged from .4 to .7. Furthermore, these effect sizes reflect within-subject comparisons of the overall activation of task versus baseline: When assessing differences in activation between groups, effect sizes can be expected to be smaller than this.

The second issue is that problems arise when there is a failure to appreciate that $p$ values are only interpretable in the context of a hypothesis testing study (de Groot, 2014). Our knowledge is still limited, and many studies in this area are exploratory: Insofar as there is a hypothesis, it is often quite general, namely, that there may be a significant association between one of the genotypes examined and one or more phenotypes. Spurious findings are likely if there are many possible ways of analyzing findings, and the measures or analyses are determined only after inspecting the data (Vul \& Pashler, 2012). This leads to the twin problems of $p$ hacking (selecting and modifying analyses until a "significant" $p$ value is found) and hypothesizing after results are known (Kerr, 1998), both of which render $p$ values meaningless. These practices are common but not easy to detect, although they may be suspected when there are numerous $p$ values just below a "significance" threshold (Simonsohn, Simmons, \& Nelson, 2015), or when the selection of measures or analyses has no obvious justification. One solution is to adopt a two-stage approach, where an association observed in an initial exploratory study (the "discovery" sample) is then tested in a more focused study that aims to replicate the salient findings in a fresh sample (the "replication" sample). This approach is now common in GWAS, after early association studies were found to produce numerous false-positive findings. Before the advent of GWAS, the majority of reported associations did not replicate consistently (Sullivan, 2007). Most genetics journals now require that, to be published, associations have to be replicated (e.g., Behavior Genetics; Hewitt, 2012), and researchers have learned that large samples are needed to obtain adequate statistical power for replication (Lalouel \& Rohrwasser, 2002) because initial reports overestimate true effect size. However, outside of GWAS, the importance of adequately powered replication is not always appreciated. As Poldrack et al. (2017, p. 117) noted, imaging genetics is "a burgeoning field that has yet to embrace the standards commonly followed in the broader genetics literature."

An alternative approach to replication is to perform a statistical correction for the number of comparisons in an analysis. However, for this to be effective, the adjustment must be made for the multiplicity of potential analyses at several levels. Consider, for instance, a study where three SNPs are studied for association with measures of neural connectivity based on four brain regions. If the SNPs are in linkage equilibrium (i.e., not associated) and the connectivity measures are uncorrelated, then it might seem that we could adequately control Type I error by using a Bonferroni-corrected $p$ value of $.05 /(3 \times 4)=.004$. However, suppose the researchers also study connectivity between brain regions, then there are six measures to consider ( $\mathrm{AB}, \mathrm{AC}, \mathrm{AD}, \mathrm{BC}, \mathrm{BD}, \mathrm{CD})$. They may go on to test two models of genetic association (dominant and recessive) and further subdivide the sample by sex, increasing the number of potential comparisons to $3 \times 6 \times 2 \times$ $2=72$ and the Bonferroni-corrected $p$ value to .0007 . Furthermore, we cannot compute this probability correctly unless all conducted tests are reported: If the authors remove reference to SNPs, genetic models, subgroups, or phenotypes that did not yield significant results, then reported $p$ values will be misleading. In GWAS, the finite search space (essentially the likely number of functional genetic variants in the human genome, estimated as around one million) means that a $p$ value threshold corrected for all possible tests can be calculated-in these studies, genome-wide significance for a single trait is typically set at $5 \times 10^{-8}$ (Sham \& Purcell, 2014).

Journal editors are becoming aware of problems of reproducibility in the field of neuroscience (Nicolas, Charbonnier, \& Oliveira, 2015), many of which are reminiscent of earlier problems in the candidate gene era (Flint, Greenspan, \& Kendler, 2010). The current study was designed to evaluate the extent to which these problems currently affect the field of human neurogenetics and to identify instances of good practice that might suggest ways of overcoming the methodological and logical difficulties that researchers in this area face.

\section{STUDY PROTOCOL}

The protocol for this study was registered on Open Science Framework (osf.io/67jwb/). Many modifications 
were subsequently made in the course of collating studies for analysis, because articles or reported measures did not readily fit into the categories we had anticipated. Furthermore, the complexity of the methods used in many studies was such that it took substantial time to identify basic information such as effect sizes, which led to us focusing on a more restricted set of study features than we had originally planned. In addition, we added Cyril Pernet to the study team, as it became clear that we needed additional expertise in neuroimaging methods to evaluate some of the articles. Departures from the protocol are noted below, with an explanation of each one.

\section{Electronic Search Strategy}

The search was conducted using the Web of Science database. We started with the 20 most highly ranked journals in neuroscience and behavior (source: https://www. timeshighereducation.com/news/top-20-journals-inneuroscience-and-behaviour/412992.article). We then excluded journals that have a wide scope of subject matter (e.g., Nature, Proceedings of the National Academy of Sciences) and those that focus on review articles (e.g., Current Opinion in Neurobiology), which left 12 suitable journals to be used for the literature search. All of these publish articles in English only.

In our protocol, we planned to examine 50 publications, but we had underestimated the amount of time needed to extract information from articles, many of which were highly complex. When this became apparent, we decided that our resources would allow us to examine 30 publications in full, and so we restricted consideration to the most recent articles, starting with June 2016 and working backwards until 30 suitable articles were selected (initial search June 2016 to June 2011).

To identify relevant articles, the names of the 12 journals were coupled with topic-specific search terms. We limited the search to studies of humans and used the following key terms:

(Nature Neuroscience OR Neuron OR Annals of Neurology OR Brain OR Molecular Psychiatry OR Biological Psychiatry OR Journal of Neuroscience OR Neurology OR Journal of Cognitive Neuroscience OR Pain OR Cerebral Cortex OR Neurolmage) AND TOPIC: (genetic OR gene OR allele) AND TOPIC: (association) AND TOPIC: (cognition OR behaviour OR individual differences OR endophenotype) AND TOPIC: (human)

\section{Selection Criteria and Data Extraction}

The first author screened abstracts found by the electronic search to identify relevant articles. The first and last author independently coded the first 500 articles and discussed sources of disagreement. This led to some refinement of the inclusion and exclusion criteria that had been specified in the original protocol, as described below (see

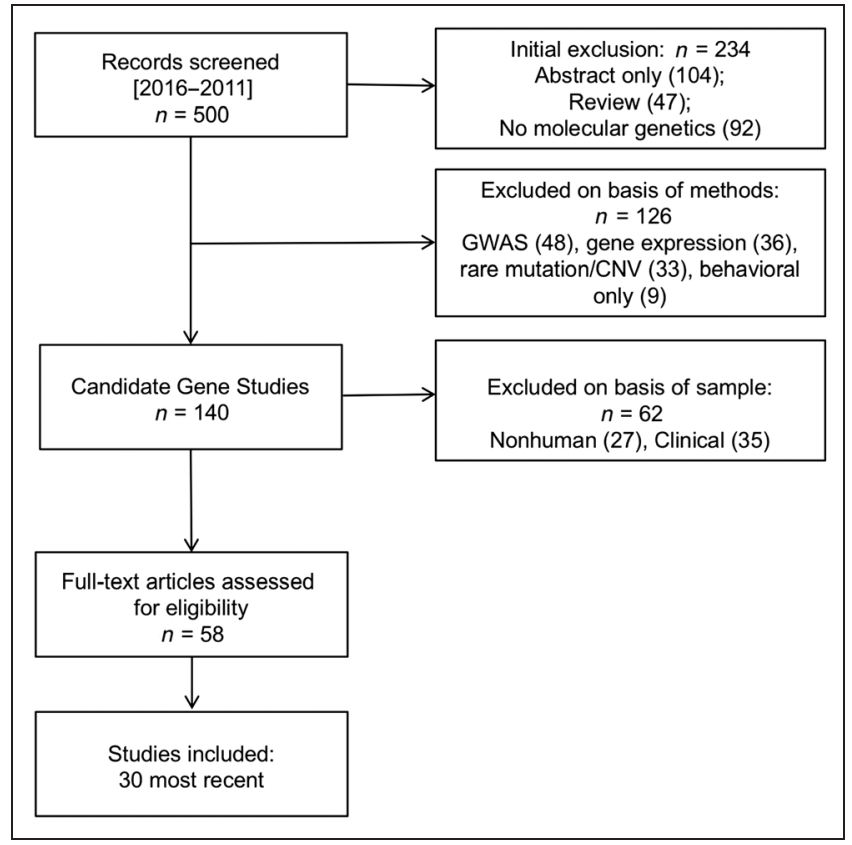

Figure 1. Flowchart showing stages of article selection.

Figure 1). The first 30 articles that met the final inclusion and exclusion criteria were fully reviewed, and metadata were extracted (see below for details).

\section{Inclusion Criteria}

- Candidate gene(s) study.

- Studies predominantly focusing on healthy individuals. This includes population-based studies that may include individuals suffering from a disorder but where the phenotype of interest is a cognitive, behavioral, or neuroimaging characteristic.

\section{Exclusion Criteria}

Original exclusion criteria specified in our protocol were as follows:

- review articles;

- GWAS;

- studies predominantly focusing on genetic associations where the phenotype is a disease or disorder (e.g., neurodegenerative disease, neurodevelopmental disorder, or psychiatric disorders).

Additional exclusionary criteria included after assembling the pool of potential studies:

- $\quad$ studies reporting an abstract only;

- $\quad$ studies solely on nonhuman species;

- studies solely focused on rare variants (i.e., those with a minor allele frequency less than 1\%, or copy number variants), because our focus was on common variation rather than disease, and rare variants and copy number variants require a different analytic approach; 
- studies focused solely on gene expression;

- studies with no molecular genetic content (e.g., twin studies);

- analyses using polygenic risk scores.

Data were extracted for the following characteristics:

1. Information about the study sample (the aim was to record information that made it possible to judge whether this was a clinical or general population sample, and if general population, whether a convenience sample or more representative);

2. all SNPs that were tested;

3. all measures of cognition, behavior, or neurological structure or function that were used as dependent variables;

4. sample size;

5. analysis method(s);

6. any results given in terms of means and variance ( $S D$ or $S E$ ) on dependent measures in relation to genotype;

7. statistics that could be used to obtain a measure of association (odds ratios, regression coefficients, $p$ values, etc.).

In our original protocol, we had planned also to evaluate the functionality of polymorphisms, to look for information on the reliability of phenotypes, and to evaluate the comprehensiveness of the literature review of each study, but the challenges we experienced in extracting and interpreting statistical aspects of the main results meant that we did not have sufficient resources to do this.

The information that we extracted was used to populate an Excel template for each study, which included information on sample size, corrections for multiple comparisons, and whether or not a replication sample was included. The sample size was used to compute two indices of statistical power using the pwr package in R (R Core Team, 2016): (i) the effect size ( $r$ ) detectable with $80 \%$ power and (ii) the power of the study to detect an effect size $(r)$ of .1 .

We planned also to extract an effect size for each study, indicating the strength of genetic influence on the phenotype of interest. This proved difficult because many studies reported a complex range of results, with some including interaction effects as well as main effects of genotype. In addition, for studies reporting neuroimaging results, large amounts of data with spatial and temporal dependencies pose considerable challenges when estimating effect sizes, and so such studies were flagged as they often required alternative approaches.

To make the task of synthesizing evidence more tractable, we identified a "selected result" for each study. To facilitate comparisons across studies and avoid the need for subjective judgment about the importance of different results, we identified this as the genotypic effect with the largest effect size (excluding any results from nonhuman species): This means that our estimates of study power give a "best case scenario." It also meant that, in our summary template, study findings were often oversimplified, but we included a "comments" field that allowed us to describe how this selected result fitted into the fuller context of the study. Our approach to computing a standard effect size is detailed below in the section on Analytic Approach.

In a further departure from our original protocol, we sent the template for each study to the first and last authors with a request that they scrutinize it and correct any errors, with a reminder sent 2-3 weeks later to those who had not responded. Acknowledgement of the email was obtained from authors of 23 of 30 studies (77\%), 19 of whom (63\%) provided the requested information, either confirming the details in the template or making suggestions or corrections. The latter were taken into consideration in the summary of each study. We initially referred to the selected result with the largest genetic effect as a "key result," and several authors were unhappy with this, as they felt that we should focus on the result of greatest interest, rather than largest effect size. We dealt with this by rewording and adding further explanation about other results in the study, noting when the selected result did not correspond to the author's main focus.

\section{Simulations}

We had not planned to include simulations in our protocol, but we found it helpful to write scripts to simulate data to explore two issues that arose. First, we considered how the false-positive rate was affected when all three models (additive, dominant, and recessive) were tested in the same data set. Second, we considered how the use of a selected sample (e.g., high-ability students) might affect genetic associations when cognitive phenotypes were used.

\section{Effect Size Estimation}

For each study, we aimed to extract an effect size, representing the largest reported effect of a genotype on a phenotype. For simple behavioral/cognitive phenotypes, it was usually possible to compute an effect size in terms of the correlation coefficient $r$, which when squared provides the proportion of variance accounted for by genotype. The correlation coefficient is identical to the regression coefficient $\beta$, when both predicted variable $(y=$ phenotype of interest $)$ and predictor $(x=$ genotype) are standardized. For a standard additive genetic model with three genotypes ( $a a, a A$, and $A A$ ), the number of "risk" alleles is the independent measure, so the regression tests for a linear increase in phenotypic score from $a a$ to $a A$ to $A A$. Where authors reported an unstandardized regression coefficient, $b$, the correlation coefficient $r$ was obtained by the formula $r=b \cdot s_{x} / s_{y}$, where $s_{x}$ and $s_{y}$ are the standard deviation for $x$ ( $N$ risk alleles) and $y$ (phenotype). Formulae from Borenstein, Hedges, 
Higgins, and Rothstein (2009) were used to derive values of $r$ when data were reported in terms of Cohen's $d$, odds ratios, or means and standard deviations by genotype. Where standard errors were reported, these were converted to standard deviations by the formula $S D=$ $S E \times \operatorname{sqrt}(N)$.

Two studies used structural equation modeling of relationships between variables, demonstrating that model fit was improved when genotype was incorporated in the model. In these cases, standardized parameter estimates or Pearson correlation coefficients relating genotype to phenotype were used to provide a direct measure of effect size $(r)$.

For studies using phenotypic measures based on neuroimaging, an effect size can be estimated if an average measure of structure (e.g., gray or white matter volume) or function (e.g., BOLD response) was taken from a brain region that was predefined in a way that was independent of the genetic contrast. For instance, the focus may be on a region that gave a significant group difference in a prior study or the region may be chosen because it reliably gives strong activation on a task of interest. If several such regions are identified, then it is necessary to correct for multiple testing (see below), but the measure can be treated like any other phenotype when computing a standardized effect size, for example, using the slope of the regression for three genotype groups in an additive model to quantify how much variance in the neuroimaging measure is accounted for by genetic differences.

Few neuroimaging studies, however, adopted that approach. More commonly, they reported peak voxel statistics. This involves a statistical approach of searching for the voxel or cluster of voxels that gives the strongest effect, sometimes in a confined ROI, sometimes over many brain regions, and sometimes over the whole brain. The search volume can consist of tens or even hundreds of thousands of voxels. It is well recognized in this field that correction of alpha levels needs to be made to control the rate of false positives, and a range of methods has been developed for this purpose. ${ }^{1}$

Although these methods make it possible to identify patterns of neural structure or function that differ reliably between groups, it is still not possible to derive a meaningful measure of effect size. This is because the focus is on just the subset of voxels that reached significance. As Reddan, Lindquist, and Wager (2017) put it, "It is like a mediocre golfer who plays 5,000 holes over the course of his career but only reports his 10 best holes. Bias is introduced because the best performance, selected post hoc, is not representative of expected performance." In addition, the extent of the overestimate will depend on study-specific variables, such as the number of voxels considered and the size of clusters. Estimates of effects will also be distorted because of spurious dependencies in the data between true effects and noise (Kriegeskorte, Simmons, Bellgowan, \& Baker, 2009). These problems are compounded by two further considerations. First, groups in genetic analyses are often unequal in size; where the dependent measure represents peak activation, the group with the biggest sample size and/or smaller variance in space will have a greater impact on the results. To continue the golfing analogy, if we compared two golfers on the basis of their best 10 games and one had played 100 games and the other only 20, then the one with the more games would look better, even if in fact there was no difference in skill.

It is not uncommon for researchers to use measures of peak activation but treat the resulting measures like more classic dependent variables (e.g., graphing means and standard errors for measures of activation across genetic groups and reporting these along with corrected $p$ values). Such estimates are inaccurate and possibly inflated, yet often these are the only kind of data available. Accordingly, where such approaches were adopted, we used the reported data to derive a "quasi-effect size," deriving $r$ from means and standard deviations, but we treated these separately from other effect sizes, as they are likely to be distorted, and it is not possible to estimate by how much.

\section{Analytic Approach}

Our analysis was predominantly descriptive and involved documenting the methodological characteristics of the 30 studies. In addition, we considered how effect size related to statistical power and the methods used to correct for multiple comparisons.

\section{RESULTS}

The genes and phenotypes that were the focus of each study are shown in Appendix 1, and full summary findings for each of the 30 studies are shown in Appendix 2 (both available via Open Science Framework: osf.io/ pex6w). These are based on the templates that were sent to the authors of the articles, but they have been modified on the basis of further scrutiny of the studies. In a preliminary check, we compared these articles to the set of 548 studies from the Neurosynth database that had been used by Poldrack et al. (2017) to document trends in sample size for neuroimaging articles between 2011 and 2015. There was no overlap between the two sets.

\section{Effect Size of Selected Result in Relation to Sample Size}

All the studies under consideration reported $p$ values, but only four explicitly reported conventional effect sizes (one as Cohen's $d$ and three as regression coefficients). Some fMRI studies mentioned "effect size" or "size of effect" when referring to brain activation, but this was on an arbitrary scale and therefore difficult to interpret. Nevertheless, we were able to compute an effect size from 
reported statistics for all studies that used behavioral (including cognitive) phenotypes and quasi-effect size (see above) for eight studies using neuroimaging phenotypes.

As noted, effect sizes of common genetic variants on behavioral or neurological phenotypes are typically small in magnitude. Where a research literature includes underpowered studies, effect size may be negatively correlated with sample size, reflecting the fact that small effects do not reach statistical significance in small samples and tend not to be published. This effect was apparent in the 30 articles included in our review. The relevant data are shown in Figure 2, where $r$ is plotted against log sample size. Quasi-effect sizes from neuroimaging studies are likely to be inflated, and so these are shown using different symbols.

The correlation between effect size and log sample size is -.85 (bootstrapped 95\% CI $[-.68, .94]$ ) for the whole sample and -.77 (bootstrapped 95\% CI $[-.38,-.94]$ ) when 10 neuroimaging studies with quasi-effect sizes are excluded. It is clear from inspection that effect sizes ( $r$ greater than .3 are seen only in studies where the total sample size is 300 or less. Only one study with a sample size of 500 or more obtained an effect size of greater than .2. The largest reported effect size mostly clustered around the line corresponding to the effect detectable with $80 \%$ power: This makes sense insofar as studies are published only if they report statistically significant results. Thus, it is not that smaller studies show larger effects, but rather than in smaller studies, small effects would not be statistically significant and so would tend to go unreported.

\section{Corrections for Multiple Comparisons}

The need to take multiple comparisons into account appears to be generally recognized: 23 of the 30 studies (77\%) made some mention of this, although they varied widely in how they handled this issue. We had originally intended to simply report the number and nature of corrections used for multiple comparisons. However, this too proved complicated because there were many ways

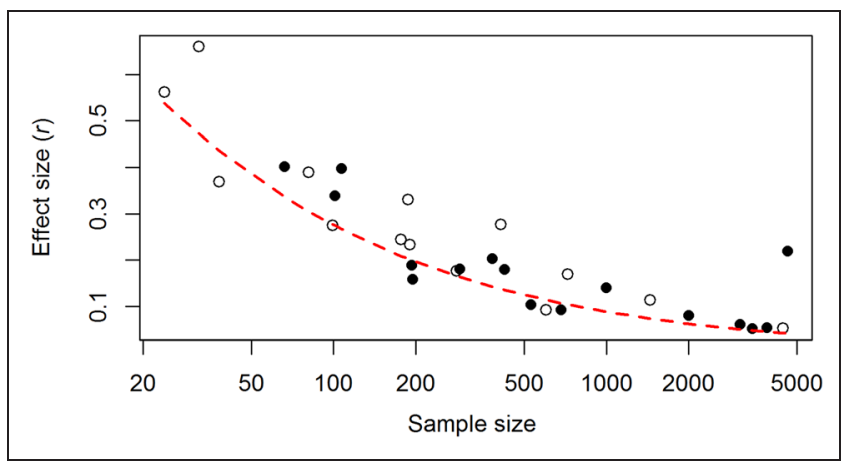

Figure 2. Largest obtained effect size in relation to sample size (on log scale). Quasi-effect sizes (see text) shown as unfilled symbols. The red dotted line shows smallest effect size detectable with $80 \%$ power. in which analytic flexibility could be manifest, with multiple comparison issues arising at several levels: in terms of analysis of subsamples, number of genetic models, number of polymorphisms, number of phenotypes, and, for neuroimaging studies, number of brain regions considered. In Table 1, we show for each study the numbers of comparisons at each level, as well as "All Comparisons," which is the product of these. Matters are more complicated when there are dependencies between variables of a given type, as discussed in more detail below. Furthermore, it could sometimes be difficult to summarize the information, if certain phenotypes were assessed for just a subset of the sample or were ambiguous as to whether they were phenotypes or moderators. In what follows, we first discuss multiplicity in terms of subgroups, then at genetic and phenotypic levels, before finally considering multiple comparisons in the context of neuroimaging studies.

\section{Subgroups}

In subgroup analysis, the association between genotype and phenotype is conducted separately for each subgroup (e.g., male and female). Typically, this is in addition to analysis of the whole sample with all subgroups included. Subgroup analysis is different from replication, where an association discovered in one sample is then confirmed in another, independent sample (see below). Most studies did not conduct any subgroup analysis, but four subdivided the participants by sex, one by ethnic group, one by age band, and one by psychiatric disorder in relatives.

It is well known that deciding to analyze subgroups after looking at the data inflates Type I error (Naggara, Raymond, Guilbert, \& Altman, 2011), but there may be good a priori reasons for distinguishing subgroups. Subsampling by sex is justified where a relevant polymorphism is located on a sex chromosome or where there are sex differences in the phenotype. Subsampling by ethnicity is generally advised to avoid spurious associations arising because of different proportions of polymorphisms in different ancestral groups (Tang et al., 2005) known as population stratification. Nevertheless, subsamples will be smaller than combined samples, so power of the analysis is reduced, and furthermore, each subsample included in an analysis will increase the likelihood of Type I error unless the alpha level is controlled. Only two of the seven studies of subgroups made any adjustment for the number of subgroups.

\section{Genetic Variation}

For the genotype part of genotype-phenotype association, there are two factors to take into account: (a) the number of polymorphisms considered and (b) the number of genetic models tested. 


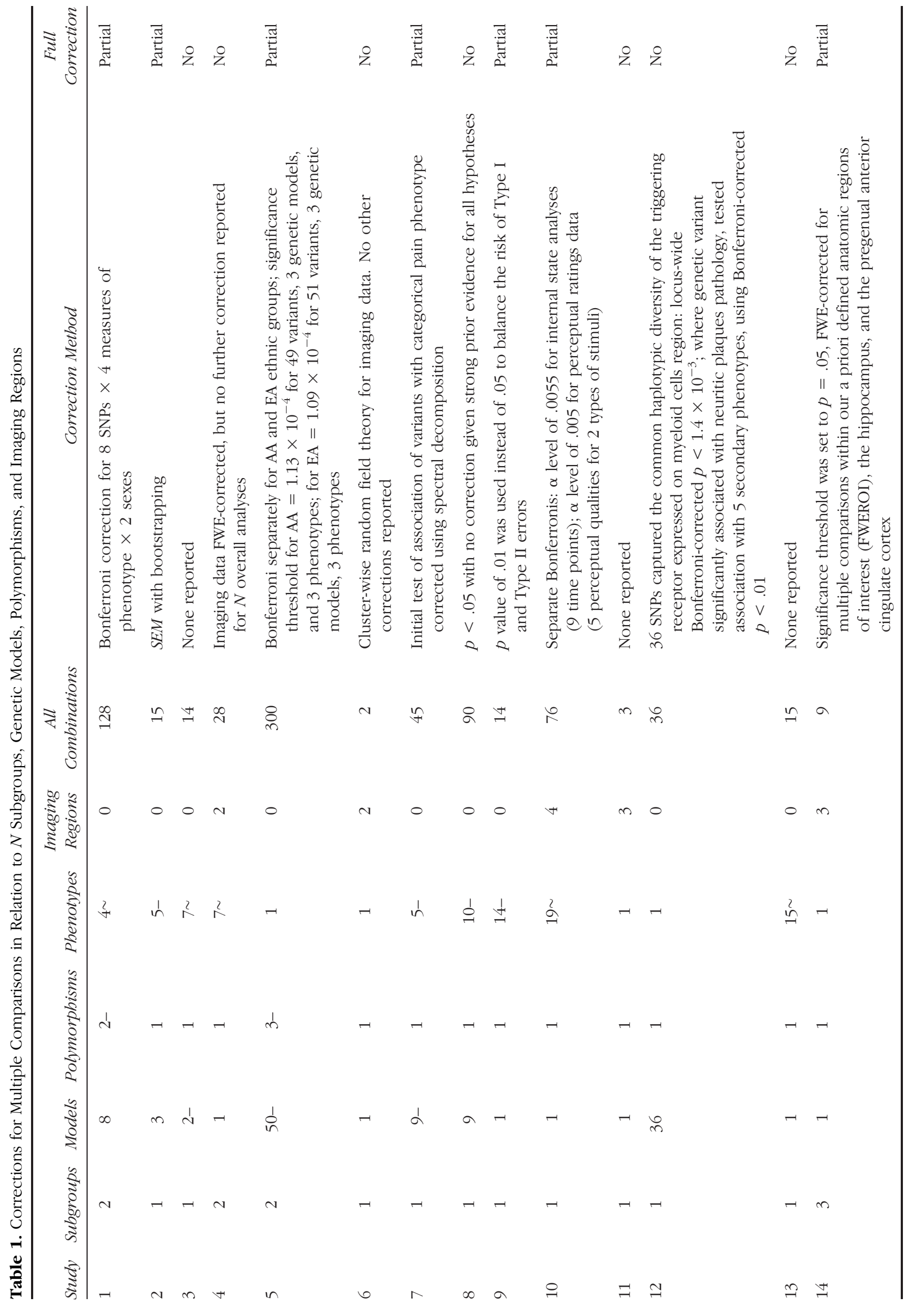




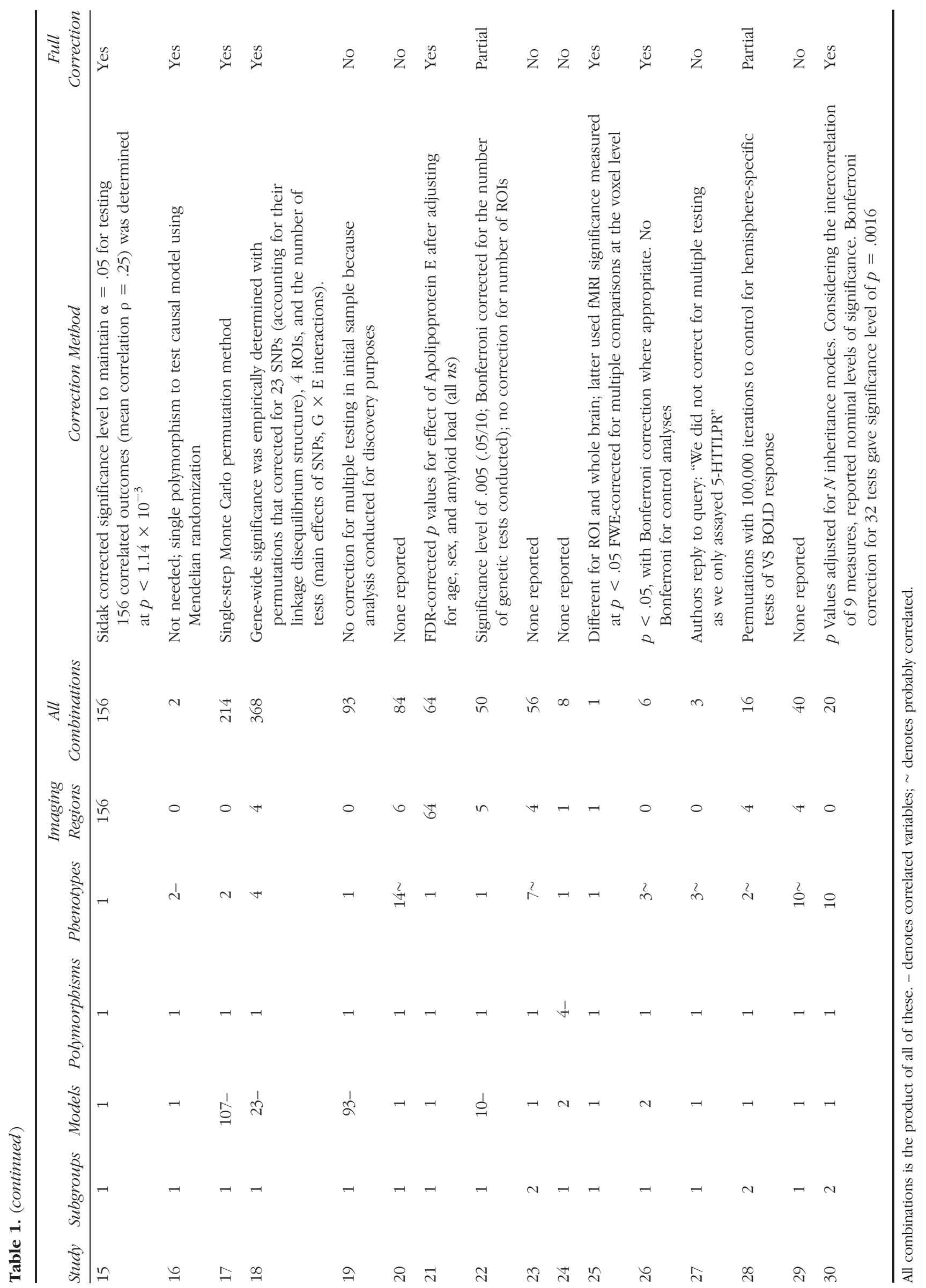

32 Journal of Cognitive Neuroscience

Volume 30, Number 1 
Number of polymorphisms. Polymorphisms are segments of DNA that take different forms in different people. ${ }^{2}$ Most studies in our analysis investigated how phenotypes related to variation in one or more SNPs, with the number of SNPs ranging from 1 to 192.

Correlation between alleles at two or more genetic loci is referred to as linkage disequilibrium. This can arise when loci are close together on a chromosome and so not separated by recombination events during meiosis, or it may be a consequence of population stratification, for example, if certain genotypes are correlated with ethnicity or if there is assortative mating. Genetic variants that are inherited together on the same chromosome (i.e., from the same parent) give rise to combinations of alleles known as haplotypes. Rather than studying SNPs, some studies categorized participants according to haplotype status; this involves looking at the sequence of DNA in longer stretches of DNA, taking parent of origin into account.

Where polymorphisms are independent, a Bonferroni correction may be used by simply dividing the critical $p$ value by the number of SNPs (Clarke et al., 2011). For polymorphisms in linkage disequilibrium, the Bonferroni correction is overly conservative. A range of methods has been developed to handle this situation, and some of these are routinely output from genetic analysis software. For instance, the dimensionality of the data may be reduced by spectral decomposition or by basing analysis on haplotype blocks rather than individual SNPs: These methods of data reduction are often incorporated as an additional step of correction for the effective number of comparisons once the dimensionality has been reduced. Clarke et al. (2011) noted that permutation methods are often regarded as the gold standard for correcting for multiple testing, but they are computationally intensive. Table 2 shows the different methods encountered in the 13 studies that reported analysis of more than one polymorphism. It is clear there is a wide variation in the types of correction that are used, and some studies do not report any correction despite studying two or more independent genotypes. Furthermore, correlations between polymorphisms were not always reported: In such cases, it was assumed they were uncorrelated.

The majority of studies $(n=17)$ did not require any correction as only one SNP was reported. It is, of course, not possible to tell whether researchers tested a larger number of variants and selectively reported only those that reached statistical significance. A problem for the field is that it is difficult to detect this practice on the basis of published results. We know that dropping nonsignificant findings is a common practice in psychology (John, Loewenstein, \& Prelec, 2012), and we may suspect selective reporting in studies where the choice of SNP seems arbitrary and unrelated to prior literature. We note below that requiring authors to report explicitly on whether all conducted tests were reported would
Table 2. Correction for Multiple Testing in Relation to Genetic Variants Considered: 13 Studies with Two or More Polymorphisms

\begin{tabular}{|c|c|c|}
\hline & $\begin{array}{c}\text { Correlated }^{a} \\
\text { Polymorphisms }\end{array}$ & $\begin{array}{c}\text { Uncorrelated } \\
\text { Polymorphisms }\end{array}$ \\
\hline No & 0 & 2 \\
\hline Bonferroni & 2 & 2 \\
\hline Data reduction $^{b}$ & 3 & 0 \\
\hline Permutation & 2 & 1 \\
\hline
\end{tabular}

${ }^{a}$ Treated as correlated if authors reported greater than chance association between SNPs.

${ }^{\mathrm{b}}$ For example, using spectral decomposition to reduce dimensionality of data or haplotype analysis

ameliorate the situation. Furthermore, study preregistration will remove uncertainty about which analyses were planned.

Eleven of the 13 studies that reported on two or more SNPs corrected for the number of genotypes tested, though two studies appeared to overcorrect, by using a Bonferroni correction for correlated SNPs. The remaining studies used a range of approaches, some of which provided useful examples of how to deal effectively with the issue of multiple testing, as described further in the Discussion.

Genetic models. Consider a polymorphic SNP, with a major (more common) allele $A$ and a minor (less common) allele $a$, giving three possible genotypes, $A A, A a$, and $a a$. Let us suppose that $A$ is the risk allele (i.e., associated with less optimal phenotype). There are three models of genetic association that are commonly tested: (i) additive model, tested by assessing the linear regression of phenotype on number of copies of allele $A$; (ii) a dominant effect, where $a a$ differs from $A A$ and $A a$, with no difference between the latter two genotypes; and (iii) a recessive effect, where $A A$ differs from $A a$ and $a a$ (see Figure 3).

Some studies considered all three types of model, whereas others tested just one type of model. In other cases, the comparison was between two genotypes that corresponded to groups identified by the length of tandem repeats, rather than base changes, and in one case a polymorphism on the $\mathrm{X}$ chromosome was considered in men, which gave a two-group comparison (Base A vs. Base $\mathrm{G}$ )—because men have only one $\mathrm{X}$ chromosome.

There was only one study that explicitly tested three genetic models for each of several SNPs (additive, dominant, and recessive), and that study included a Bonferroni correction to adjust for this. This is, in fact, overly conservative, as the predictions of an additive model partially overlap with those of recessive and dominant models. We devised a simulation to evaluate this situation. The phenotype was modeled as a random normal 
Figure 3. Schematic of three types of genetic model.

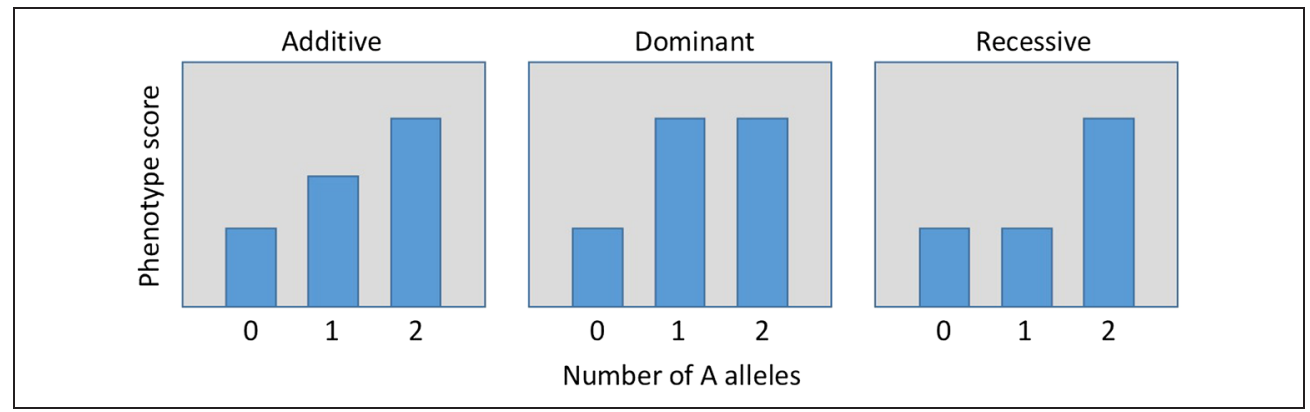

deviate, unrelated to simulated alleles at two loci for an SNP (A or a), so odds of obtaining a $p$ value of $<.05$ for any one analysis should be 1 in 20 . Regression analyses were run to look for an effect of number of A alleles (additive model), the effect of AA + Aa versus aa (dominant model), and the effect of $\mathrm{AA}$ versus $\mathrm{Aa}+$ aa (recessive model). Results indicated that adequate control for multiple comparisons is obtained by dividing the $p$ value by two (Figure 4). One study focused on interactions between two loci (epistasis) rather than main effects. Of the 28 remaining studies reporting just one genetic contrast per polymorphism, 17 reported results from additive genetic models (contrasting those with zero, one, or two copies of an allele), nine reported only nonadditive (dominant or recessive) models, and two included a mixture of additive and nonadditive models, depending on the SNP. Of those reporting nonadditive models, some justified the choice of model by reference to previous studies, but others grouped together heterozygotes and homozygotes with the minor allele for convenience because the latter group was too small to stand alone.

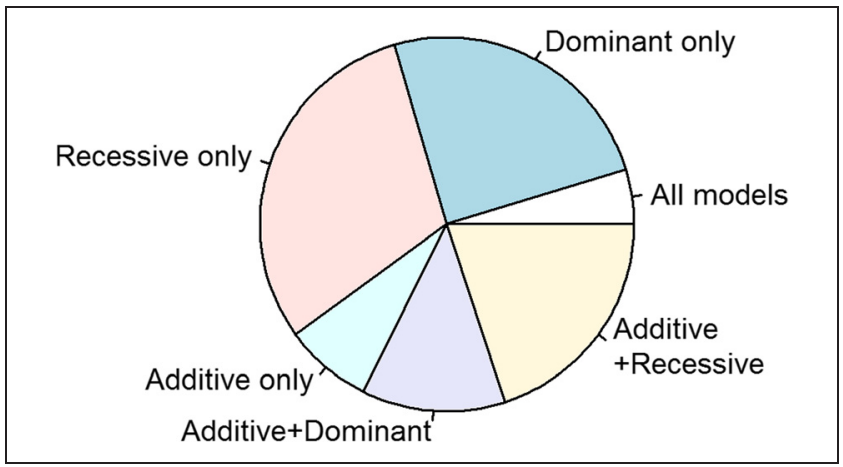

Figure 4. Simulated data showing proportions of significant $(p<.05)$ runs of a simulation that tests for all three genetic models when null hypothesis is true. The total region of the pie corresponds to $10 \%$ of all runs (i.e., twice the expected 5\%, but lower than the 14\% that would be expected if the three models were independent). Note that we seldom see runs where both dominant and recessive models are significant, because they lead to opposite patterns of association (Figure 3), but it is not uncommon to see runs where both additive and recessive, or additive and dominant models are significant. For simulation code, see osf.io/4dymh.

\section{Phenotypes}

Phenotypes included measures of cognition, behavior, psychiatric, or brain functioning. For neuroimaging studies, the phenotypes included measures of brain structure or activation in response to a task. As described more fully below, the neuroimaging literature has developed particular strategies for dealing with the multiple contrasts issue; in Table 1, the number of brain regions is ignored when documenting the number of phenotypes. However, if brain activation was measured in several different tasks, then each task corresponded to a phenotype as defined for our purposes.

The simplest situation was where a phenotype was assessed using a behavioral or cognitive test that yielded a single measure, but this type of study was rare. Multiple phenotypic measures were common. As with genotypes, these were frequently correlated with one another, making Bonferroni correction too conservative, but studies often failed to report the extent of correlation between phenotypes. Often multiple measures were used to test the same construct, and so it is to be expected they would be intercorrelated: In such cases, if no mention is made of extent of intercorrelation, we record the correlation as "unclear" in Tables 1 and 3. There was wide variation in the corrections used for the number of phenotypes. No correction was reported for 11 of 19 studies (58\%) that included two or more phenotypes (see Table 3). In all cases, the phenotypes were correlated (or probably correlated)—-thus, conventional Bonferroni correction would have been too stringent.

Of the four studies using Bonferroni correction, three had correlated phenotypes, but one (Study 9) took into account correlation between variables by reducing the denominator in the correction, though in what appeared to be an arbitrary fashion. More complex methods using permutation or bootstrapping were used in only three studies.

\section{Neuroimaging Phenotypes}

In neurogenetics, the goal is to find structural or functional correlates of genotypes. It has long been recognized that neuroimaging poses multiple comparison problems of its 
Table 3. Correction for Multiple Testing in Relation to whether Behavioral Phenotypes Are Correlated

\begin{tabular}{lcccc}
\hline & NA & Correlated & $\begin{array}{c}\text { Probably } \\
\text { Correlated }\end{array}$ & Uncorrelated \\
\hline None & 0 & 2 & 9 & 0 \\
Bonferroni & 0 & 1 & 2 & 1 \\
Permutation & 0 & 1 & 0 & 2 \\
Not needed & 11 & $1^{\mathrm{a}}$ & 0 & 0 \\
\hline
\end{tabular}

${ }^{\mathrm{a}}$ Mendelian randomization method.

own, because it typically involves gathering information from tens if not hundreds of thousands of voxels, corresponding to gray or white matter derived variables in the case of structural imaging (e.g., volume, thickness, anisotropy) or to proxies for underlying neural activity or connectivity in functional imaging. The spatial and temporal dependencies between voxels need to be taken into account.

The selection of an ROI is key. The commonest approach is to do a whole-brain analysis. Some studies in our review selected specific regions, and some assessed more than one region: In such cases, it is not sufficient to do statistical adjustments within the region-one still needs to treat each region as a new phenotype, with further correction applied to take the potential Type I error inflation into account. The numbers for neuroimaging regions shown in Table 1 refer to the total ROIs that were considered in the analysis.

For the current analysis, we categorized neuroimaging articles according to whether they used an ROI specified a priori on the basis of previous research, with activation compared between genotype groups within that whole region. In such a case, it is possible to compare activation across genotypes to get a realistic effect size. However, as noted above, where the analysis involves first finding the voxel or cluster within an ROI that gives peak activation and then comparing groups, it is not possible to accurately estimate effect sizes, because the method will capitalize on chance and so inflate these. Studies that adopted this approach are therefore flagged in Figure 1 as giving a "quasi-effect size."

\section{Replication Samples}

We had originally intended to classify studies according to whether they included a replication sample, but this proved inadequate to handle the different approaches used in our collection of studies. As noted by Clarke et al. (2011), a true replication uses the same SNPs and phenotypes as the original study, but in practice replication studies often depart from such fidelity and may study nearby variants of the same gene or alternative measures of the phenotype. We categorized the replication status of each study as follows:

(a) Study includes a full replication using comparable genotypes and phenotypes in the discovery and replication samples. This classification was less straightforward than it may appear. Consider, for instance, Study 1: The replication sample included the same SNPs and measures from one of the same questionnaires as used in the discovery sample, but with a slightly different subset of items. In general, we treated a replication as full provided the measures were closely similar, so a case such as this would be regarded as a full replication.

(b) Study includes a partial replication, but with some variation in genotypes or phenotypes in the discovery and replication samples.

(c) Study is a direct replication of a previous study, so no replication sample is needed.

(d) Study does not set out to replicate a prior study (though choice of phenotypes and genotypes is likely to be influenced by prior work) and does not include a replication sample.

Even with this classification scheme, categorization was not always straightforward. For instance, studies that did not include a replication sample would nevertheless usually aim to build on prior literature and might replicate previous findings. These were categorized as "prior" (option b) only if they were explicitly described as aiming to replicate the earlier work. We anticipated that replication samples would be more common in journals that regularly published genetics articles, where the need for replication is part of the research culture. Table 4 shows the number of articles according to replication status and journal. Note that there were three journals in our search for which no articles met our inclusion criteria in the time window we used: Nature Neuroscience, Neuroimage, and Brain.

Table 4. Number of Studies including Replication Sample, by Journal

\begin{tabular}{lcccc}
\hline & Yes & Partial & Prior $^{a}$ & No \\
\hline Annals of Neurology & 0 & 0 & 0 & 1 \\
Biological Psychiatry & 2 & 1 & 0 & 4 \\
Cerebral Cortex & 0 & 0 & 0 & 1 \\
Journal of Cognitive Neuroscience & 0 & 1 & 0 & 2 \\
Journal of Neuroscience & 0 & 1 & 0 & 2 \\
Molecular Psychiatry & 4 & 1 & 2 & 4 \\
Neurology & 0 & 0 & 0 & 1 \\
Neuron & 0 & 0 & 0 & 1 \\
Pain & 1 & 0 & 0 & 1 \\
\hline
\end{tabular}

${ }^{a}$ Study explicitly designed to replicate a prior finding. 
Figure 5. Mean $z$ scores on a phenotype for three genotypes, when the true association between genotype and phenotype in the population is $r=.2$. Data come from 10,000 runs of a simulation. The left hand panel shows the association in the full population; the middle panel shows means when the sample is taken only from those in the top $50 \%$ of the population on the phenotype measure; and the right-hand panel shows results when only the top third of the population is included. Ns are shown above the bars. As the selection becomes more extreme, the proportions of each genotype start to depart from the expected 1:2:1 ratio. The script simulating genopheno cutoffs.R is available on: https://github.com/oscci/SQING_repo.
Although the numbers are too small to be convincing, we may note that, in line with expectations, Molecular Psychiatry, which published the most studies in neurogenetics, was the journal with the highest proportion of studies including a replication, whereas neuroscience journals that did not have a genetics focus and published few genetics studies were more likely to publish studies without any replication sample.

\section{Use of Selected Samples}

Some of the studies that we evaluated used samples from the general population, some used convenience samples, and some did not clarify how the sample had been recruited. Use of students has been criticized, on the grounds that people from Western, Educated, Industrialized, Rich, and Democratic (WEIRD) societies are a very restricted demographic from which to make generalizations about human behavior (Henrich, Heine, \& Norenzayan, 2010). In the context of genetic research, however, other serious problems arise from the use of highly selected samples. Quite simply, if the phenotypic scores of a sample cover a restricted range, then power to detect genetic associations can be seriously affected.

We illustrate this with a simulation of an association between a genetic variant and a phenotype that has an effect size of $r=.2$ in the general population. Let us assume that the minor allele frequency is .5 , so the ratio of genotypes $a a, a A$, and $A A$ in the general population is $1: 2: 1$. Now, suppose we study a sample where everyone is above average on the phenotype, that is, we only include those with positive $z$-scores. As shown in Figure 5, the effect of genotype on phenotype becomes substantially weaker. If we take an even more extreme group, that is, the top third of the population, then the effect is no longer detectable in a moderate-sized sample. As also shown in Figure 5, as the association between genotype and phenotype decreases with selection, the ratio of the three genotypes changes, because those with the risk allele are less likely to be included in the sample. In fact, when there is strong selection, the effect of genotype will be undetectable, but the frequency of the three genotypes starts to depart significantly from expected values (see Figure 6).

A corollary of this effect of sample selection is that moderate effect sizes in highly selected samples are implausible when the phenotype is related to the criterion for selection. This is because a moderate effect in a selected group would entail a much larger effect size in the general population, as well as skewing of the genotype distribution in the selected sample. Sample selection

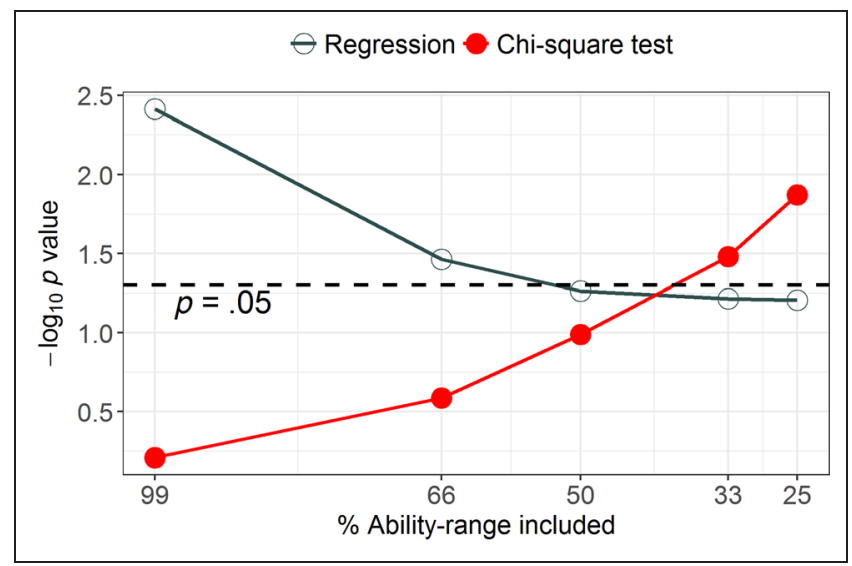

Figure 6. Relationship between genotype and phenotype depending on how participants are selected. The $-\log _{10} p$ values of the regression coefficient (black unfilled circles) are shown for the association between genotype and phenotype for data simulated as in Figure 5, depending on whether the analysis is done on the whole population or a selected subset. The significance of the association decreases as the selection becomes stricter. The dotted line shows the $\log p$ value corresponding to $p=.05$. When there is strong selection (inclusion only of top $33 \%$ or $25 \%$ of population on a phenotype $z$ score), there is significant departure from the expected 1:2:1 ratio of genotypes (as tested by chi-square test, red line). 
is therefore crucial. There may be situations when use of student samples is acceptable, because student status is unrelated to the phenotype of interest. However, where we are studying cognitive phenotypes, we stack the odds against finding associations with genotypes if we only study a high-functioning subset of the population. This can pose problems because, even when efforts are made to recruit a wide range of participants, those who volunteer tend to be biased toward more affluent and educated people (Rosenthal \& Rosnow, 1975).

\section{DISCUSSION}

This in-depth analysis of 30 studies from top neuroscience journals complements other evaluations of data quality that have used text-mining methods to extract information from larger datasets. The studies varied widely in methods and phenotypes that were studied, with some providing good examples of best practice in the field. Nevertheless, we found that when neuroscienceoriented journals publish studies that include genetic analysis, they often fail to adopt the same stringent standards for sample size and replication as have become mandatory in the genetics literature.

An important limitation of our analysis is that we evaluated only 30 highly heterogeneous studies; it would not be realistic to assume that the proportion of studies with specific characteristics is representative of the field as a whole. Nevertheless, even with this small sample, it is clear that many genetic studies with neuro or behavioral phenotypes are underpowered and/or did not correct adequately for multiple testing, even though they were published in top journals.

Another limitation of our study is that it is based on just one "selected result" per study, selected as the genetic association with the largest effect size. Many studies addressed questions that went beyond simple association between genotype and phenotype. Some considered the impact of functional groups of genes (e.g., Study 5) or looked at complex interactions between genetic variants, brain and behavior phenotypes (e.g., Study 10). A few complemented studies of humans with animal models (e.g., Study 11). We note that studies that may look inconclusive when evaluated purely in terms of one selected result can compensate for this with converging evidence from a range of sources, and our analysis is not sensitive to this.

Despite this limitation of our approach, our analysis highlighted several issues that may need to be addressed in order for neurogenetic research to fulfill its promise.

\section{Sample Size and Power}

Sample sizes in this area are often too small to detect likely effects of genetic variation, particularly when neuroimaging phenotypes are used. A similar issue was highlighted for neuroimaging studies in general by Poldrack et al. (2017), although they noted that sample sizes are now increasing as awareness of the limitations of small studies is growing. They concluded that sample sizes need to be justified by an a priori power analysis. The problem for researchers is that not only is power analysis complicated in neuroimaging (Mumford \& Nichols, 2008) but also that these studies are difficult and time-consuming to conduct and that recruitment of suitable samples can take months if not years. However, Poldrack et al. (2017, p. 117) argued: "We do not believe that the solution is to admit weakly powered studies simply on the basis that the researchers lacked the resources to use a larger sample." Instead, they recommend that, following the example of the field of genetics, researchers need to start working together in large consortia, so that adequate power can be achieved. A complementary approach is to preregister a study, so that hypotheses, methods, and analytic strategy are decided and are publicly registered before the data are collected; this can be invaluable in guarding against publication bias and the dangers of a flexible analytic pipeline. Some journals now offer Registered Reports, an approach where publication of a preregistered study is offered, conditional on satisfactory reviews and adherence to the preregistered protocol (Chambers, 2013).

An optimistic interpretation of the data in Figure 2 is that larger effect sizes are seen in smaller studies because these are studies that use highly specific measures of the phenotype that are not feasible with large samples. In particular, there is a widespread belief that neuroimaging will show stronger genetic effects than behavioral measures because it is closer to the mechanism of gene action. However, a more pessimistic interpretation is that where large effect sizes are seen in neuroimaging studies these are likely to be false discoveries arising from the use of small sample sizes with a very flexible analytic pipeline and methods that tend to overestimate effect sizes.

\section{Calculation of Effect Size}

Our analysis highlighted another problem inherent in neuroimaging studies: the difficulty of specifying effect sizes. Lakens (2013) noted that effect size computations are not only crucial for establishing the magnitude of reported effects but also for creating a literature that is useful for future researchers by providing data in a format that can be combined with other studies in a metaanalysis or which can be used to guide power calculations for future studies. Yet in neuroimaging, this is not standard. Indeed, only 3 of the 30 studies that we included explicitly mentioned effect sizes with a conventional interpretation of that term. This is consistent with a systematic review by Guo et al. (2014), who found that only 8 of 100 neuroimaging studies reported effect sizes. When reported, effect sizes are typically shown for regions with 
the strongest effects and/or at the maximum voxel, leading to biased estimates. Correcting for multiple comparisons analyses further distorts these estimates, as the strongest voxels will be those with "favorable" noise (i.e., spurious activity that adds to a true effect).

\section{Correction for Multiple Comparisons}

Most studies considered the issue of correction for multiple comparisons, but few fully corrected for all the tests conducted, taking into account the number of subgroups, genetic models, polymorphisms, and phenotypes. Researchers appear to be aware of the multiple testing problem, but there is not one good solution, and the impression was that sometimes authors thought they had done enough by applying standard corrections for fMRI and did not need to correct for other aspects of the study. For instance, studies looking at correlations between genotypes or phenotypes in ROI would have multiple comparisons procedures for whole-brain analyses but would either compute correlations for each ROI with no control or conversely adopt a Bonferroni correction (which controls exactly the Type I error rate), which is known to be overconservative.

In the field of genetics, a range of approaches has been developed for assessing associations when polymorphisms are not independent (i.e., in linkage disequilibrium); some of these, such as methods of data reduction by spectral decomposition or permutation tests, could be more widely applied (Clarke et al., 2011). For instance, extraction of latent factors from correlated phenotypes would provide a more sensitive approach for identifying genetic associations, where a range of measures is used to index a particular construct, such as anxiety or memory.

\section{Replication}

Few studies included an independent replication sample, explicitly separating out the discovery and replication phases. This approach is now standard in GWAS and has contributed to the improved reproducibility of findings in that literature. In principle, this is a straightforward solution. In practice, however, it requires additional resources and means that studies take longer to complete. It also raises the possibility that findings in the discovery phase will not be replicated, in which case the overall results may be ambiguous. One solution to this problem is to apply a more stringent alpha level at the discovery phase than at the replication phase and also to present results meta-analyzed across both phases (Lander \& Kruglyak, 1995). However, power calculations need to take into account the "winner's curse" phenomenon, which refers to the upward biasing of effect sizes when an original association emerged from a study considering many variants (Sham \& Purcell, 2014).

\section{Completeness of Reporting}

An unexpected feature of many of the studies that we analyzed was the difficulty of finding the methodological information that we required from the published articles. Because there is no standard format for reporting methods, it could be difficult to know whether specific information (e.g., whether phenotypes were correlated) was simply omitted or whether it might be found in Supplementary Material or figure legends, rather than the Methods section. Consequently, we had to read studies many times to find key information.

Most of the journals that we included had stringent length limits or page charges, which might make it difficult for authors to report all key information. Exceptions were Neuroimage, Journal of Neuroscience, Pain, and Neuron. It is noteworthy that in 2016 Neuron introduced new guidelines for structured, transparent, accessible reporting and removed any length limit on Methods (www. cell.com/star-methods), with the goal of improving reproducibility of published studies.

\section{Complexity of Analyses}

Several studies used complex analytic methods that were difficult to evaluate, despite the range of disciplinary expertise covered by the coauthors of our study. This in itself is potentially problematic for the field, because it means that reviewers will either decline to evaluate all or part of a study or will have to take analyses on trust. One solution would be for journals to require researchers to make available all analysis scripts as well as raw data, so that others could work through the analysis.

\section{Further Considerations}

We briefly mention here two additional issues that we were not able to evaluate systematically in the 30 articles that we considered but are relevant for future research in this area.

\section{Validity of Genotype-Phenotype Association}

We can be most confident that an association is meaningful if the genetic variant has been shown to be functional, with physiological effects that relate to the phenotype. Nevertheless, the ease of demonstrating functionality is much greater for some classes of variants than others. Furthermore, an association between an SNP and a phenotype does not mean that we have found a functional polymorphism. Associated SNPs often lie outside genes and may be associated with phenotypes only because they are close to relevant functional variants-what has been referred to as "indirect genotyping" (Clarke et al., 2011). Information about such variants can be valuable in providing landmarks to the key functional variant. With indirect genotyping, patterns of association may vary depending 
on samples, because different samples may have different patterns of linkage disequilibrium between genes and markers. It follows that a failure to replicate does not necessarily mean we have a false positive.

\section{Reliability and Heritability of Phenotypes}

The phenotypes that are used in genetic association studies are increasingly diverse (Flint \& Munafò, 2013). The idea behind the endophenotype concept is that a brain- based measure will be a more valid measure of the phenotypic effect of a genetic variant than other types of measure, because it is a more direct indicator of a biological effect. However, evidence for this assumption is lacking, and the strength of effects will depend on reliability as well as validity of phenotype measures. Quite simply, if a measure varies from one occasion of measurement to another, it is much harder to detect group differences even if they are real, because there will be noise masking the true effects. Therefore, it is advisable before embarking

Table 5. Key Information for Neurogenetic Studies

\section{Sample}

- Provide a power calculation to determine the sample size. The usual recommendation is for $80 \%$ power based on estimated effect size, which may be based on results with this genetic variant in previous studies. If no prior effect size is available, it is advisable to compute power with effect size no greater than $r=.2$, as few common genetic variants have effects larger than this. For neuroimaging studies, the application Neuropower (Durnez, Degryse, Seurinck, Moerkerke, \& Nichols, 2015) is a user-friendly toolbox to help researchers determine the optimal sample size from a pilot study.

- Give total sample size. Where different numbers are involved at different points in a study, a flowchart is helpful in clarifying the numbers and reasons for exclusions.

- State how the sample was recruited and whether they are representative of the general population for the phenotype of interest.

\section{Genetic variants}

- State how many genetic variants were considered in the analysis.

- List all genetic variants, regardless of whether they gave significant results.

- Give background information indicating what is known about the genetic variants, what is known about the minor allele frequency, and whether they are functional.

- State whether or not the genetic variants are in linkage disequilibrium, and if so, how this is handled in the analysis.

- State which genetic models were tested and where genotypes are combined, whether this was to achieve a workable sample size or whether the decision was based on prior research.

\section{Phenotypes}

- State whether phenotypes are known to be heritable(e.g., using evidence from twin studies).

- Provide information on the test-retest reliability of the phenotype.

- State whether phenotypes are intercorrelated.

- Neuroimaging phenotypes involved many technical choices affecting the processing pipeline. Guidelines for reporting details of neuroimaging studies have been developed with the hope of improving reproducibility. The details of analytic information go beyond the scope of this article, but useful information is given in Box 4 from Poldrack et al. (2017).

\section{Analysis}

- State which analyses were planned in advance. Post hoc analyses can be useful, but only if they are clearly distinguished from a priori hypothesis testing analysis. Where there is a clear a priori hypothesis, consider preregistering the study.

- Describe the total number of independent tests that are conducted on the data. Describe the approach used to deal with multiple comparisons, bearing in mind that other approaches exist in cases where a Bonferroni correction is likely to be overconservative.

- Make scripts for processing the data openly available on a site such as Github or Open Science Framework. It is common for authors to describe complex methods that are hard even for experts to understand. By making scripts accessible, authors make their articles easier to evaluate. Scripts can also serve as a useful training function and facilitate replication.

\section{Results}

- Do not rely solely on reporting derived statistics and $p$ values.

- Show measures of central tendency and variation for each genotype group in relation to each phenotype, together with the effect size, where it is possible to compute this. Where the phenotype is categorical, report the proportions of people with each genotype who belong in the category. 
on a genetic association study to optimize-or at least assess - reliability of phenotypic measures. Psychometric tests typically are designed to take this into account and data on reliability will be available, but for most experimental and behavioral measures, this is not the case. Furthermore, indices from functional imaging can vary from time to time (Nord, Gray, Charpentier, Robinson, \& Roiser, 2017), and even structural imaging indices are far from perfectly reliable. Further problems occur when applying methods such as fMRI to the study of individual differences where people may differ in brain structure or trivial factors, such as movement in the scanner, masking meaningful individual variation (Dubois \& Adolphs, 2016). As noted by Carter et al. (2016), neurogenetic studies rely on the assumption that the phenotype is heritable. Yet, for many of the phenotypes studied in this field, evidence is lackingusually because there are no twin studies using that specific phenotype. Heritability will be limited by reliability: A measure that shows substantial variation within the same person from one occasion to the next will not show good agreement between genetically related individuals.

\section{Proposed Reporting Requirements for Future Articles}

We conclude by making some suggestions that will make it easier for future researchers to understand neurogenetic studies and to combine these in meta-analyses, as detailed in Table 5. Ultimately, this field may need more formal reporting guidelines of the kind that have been designed to improve reproducibility of research in other areas, such as the guidelines for life sciences research introduced by Nature journals in 2015 (Nature Publishing Group, 2015) and the COBIDAS guidelines for MRI (Nichols et al., 2016). Making formal recommendations is beyond the scope of this article, but we suggest that if authors systematically reported this basic information in the Methods section of articles, it would be a major step forward.

\section{Acknowledgments}

Dorothy Bishop and Paul Thompson are supported by Wellcome Trust Programme Grant 082498/Z/07/Z. Marcus R. Munafò is a member of the UK Centre for Tobacco and Alcohol Studies, a UKCRC Public Health Research Centre of Excellence. Funding from British Heart Foundation, Cancer Research UK, Economic and Social Research Council, Medical Research Council, and the National Institute for Health Research, under the auspices of the UK Clinical Research Collaboration, is gratefully acknowledged. Support from the Medical Research Council (MC_UU_12013/6) is also gratefully acknowledged. Scripts for the analyses reported in this article are available on https:// github.com/oscci/SQING_repo. Appendices 1 and 2 are available on Open Science Framework: osf.io/pex6w.

Reprint requests should be sent to Dorothy V. M. Bishop, Department of Experimental Psychology, University of Oxford, Oxford, OX1 3UD, UK, or via e-mail: dorothy.bishop@psy.ox.ac.uk.

\section{Notes}

1. For more explanation, see Box 1 on Open Science Framework: osf.io/akuny.

2. For more explanation, see Box 2 on Open Science Framework: osf.io/akuny.

\section{REFERENCES}

Bigos, K. L., Hariri, A. R., \& Weinberger, D. R. (2016). Neuroimaging genetics. In K. L. Bigos, A. R. Hariri, \& D. R. Weinberger (Eds.), Neuroimaging genetics: Principles and practices (pp. 1-14). New York: Oxford University Press.

Borenstein, M., Hedges, L. V., Higgins, J. P. T., \& Rothstein, H. R. (2009). Introduction to meta-analysis. Wiley.

Button, K. S., Ioannidis, J. P. A., Mokrysz, C., Nosek, B. A., Flint, J., Robinson, E. S. J., et al. (2013). Power failure: Why small sample size undermines the reliability of neuroscience. Nature Reviews Neuroscience, 14, 365-376.

Carter, C. S., Bearden, C. E., Bullmore, E. T., Geschwind, D. H., Glahn, D. C., Gur, R. E., et al. (2016). Enhancing the informativeness and replicability of imaging genomics studies. Biological Psychiatry, 82, 157-164.

Chambers, C. D. (2013). Registered reports: A new publishing initiative at Cortex. Cortex, 49, 609-610.

Champely, S. (2016). pwr: Basic functions for power analysis. $\mathrm{R}$ package version 1.2-0. Retrieved from https://cran.r-project. org $/$ package $=$ pwr.

Clarke, G. M., Anderson, C. A., Pettersson, F. H., Cardon, L. R., Morris, A. P., \& Zondervan, K. T. (2011). Basic statistical analysis in genetic case-control studies. Nature Protocols, 6 , 121-133.

de Groot, A. D. (2014). The meaning of "significance" for different types of research [translated and annotated by Eric-Jan Wagenmakers, Denny Borsboom, Josine Verhagen, Rogier Kievit, Marjan Bakker, Angelique Cramer, Dora Matzke, Don Mellenbergh, and Han L. J. van der Maas]. Acta Psychologica, 148, 188-194.

Dubois, J., \& Adolphs, R. (2016). Building a science of individual differences from fMRI. Trends in Cognitive Sciences, 20, 425-443.

Durnez, J., Degryse, J., Seurinck, R., Moerkerke, B., \& Nichols, T. E. (2015). Prospective power estimation for peak inference with the toolbox neuropower. In Second Belgian Neuroinformatics Congress (Vol. 9). Frontiers Media.

Flint, J., Greenspan, R. J., \& Kendler, K. S. (2010). How genes influence behavior. Oxford: Oxford University Press.

Flint, J., \& Munafò, M. R. (2007). The endophenotype concept in psychiatric genetics. Psychological Medicine, 37, 163-180.

Guo, Q., Thabane, L., Hall, G., McKinnon, M., Goeree, R., \& Pullenayegum, E. (2014). A systematic review of the reporting of sample size calculations and corresponding data components in observational functional magnetic resonance imaging studies. Neuroimage, 86, 172-181.

Henrich, J., Heine, S. J., \& Norenzayan, A. (2010). The weirdest people in the world? Behavioral and Brain Sciences, 33, 61-83.

Hewitt, J. K. (2012). Editorial policy on candidate gene association and candidate gene-by-environment interaction studies of complex traits. Behavior Genetics, 42, 1-2.

John, L. K., Loewenstein, G., \& Prelec, D. (2012). Measuring the prevalence of questionable research practices with incentives for truth telling. Psychological Science, 23, 524-532.

Kerr, N. L. (1998). HARKing: Hypothesizing after the results are known. Personality and Social Psychology Review, 2, 196-217. 
Kriegeskorte, N., Simmons, W. K., Bellgowan, P. S. F., \& Baker, C. I. (2009). Circular analysis in systems neuroscience: The dangers of double dipping. Nature Neuroscience, 12, 535-540.

Lakens, D. (2013). Calculating and reporting effect sizes to facilitate cumulative science: A practical primer for $t$ tests and ANOVAs. Frontiers in Psychology, 4, 863.

Lalouel, J. M., \& Rohrwasser, A. (2002). Power and replication in case-control studies. American Journal of Hypertension, 15, 201-205.

Lander, E., \& Kruglyak, L. (1995). Genetic dissection of complex traits: Guidelines for interpreting and reporting linkage results. Nature Genetics, 11, 241-247.

Mumford, J. A., \& Nichols, T. E. (2008). Power calculation for group fMRI studies accounting for arbitrary design and temporal autocorrelation. Neuroimage, 39, 261-268.

Naggara, O., Raymond, J., Guilbert, F., \& Altman, D. G. (2011) The problem of subgroup analyses: An example from a trial on ruptured intracranial aneurysms. American Journal of Neuroradiology, 32, 633-636.

Nature Publishing Group. (2015). Reporting life sciences research. Retrieved from https://www.nature.com/authors/ policies/reporting.pdf.

Nichols, T. E., Das, S., Eickhoff, S. B., Evans, A. C., Glatard, T., Hanke, M., et al. (2016). Best practices in data analysis and sharing in neuroimaging using MRI. bioRxiv, 054262.

Nicolas, G., Charbonnier, C., \& Oliveira, J. R. M. (2015). Improving significance in association studies: A new perspective for association studies submitted to the Journal of Molecular Neuroscience. Journal of Molecular Neuroscience, 56, 529-530.
Nord, C. L., Gray, A., Charpentier, C. J., Robinson, O. J., \& Roiser, J. P. (2017). Unreliability of putative fMRI biomarkers during emotional face processing. Neuroimage, 156, 119-127.

Poldrack, R., Baker, C. I., Durnez, J., Gorgolewski, K., Matthews, P. M., Munafò, M., et al. (2017). Scanning the horizon: Towards transparent and reproducible neuroimaging research. Nature Reviews Neuroscience, 18, 115-126.

R Core Team. (2016). R: A language and environment for statistical computing. Vienna: R Foundation for Statistical Computing. Retrieved from https://www.r-project.org/.

Reddan, M. C., Lindquist, M. A., \& Wager, T. D. (2017). Effect size estimation in neuroimaging. JAMA Psychiatry, 74, 207-208.

Rosenthal, R., \& Rosnow, R. (1975). The volunteer subject. New York: Wiley.

Sham, P., \& Purcell, S. (2014). Statistical power and significance testing in large-scale genetic studies. Nature Reviews Genetics, 15, 335-346.

Simonsohn, U., Simmons, J. P., \& Nelson, L. D. (2015). Better $p$ curves: Making $p$ curve analysis more robust to errors, fraud, and ambitious $p$ hacking, A reply To Ulrich and Miller (2015). Journal of Experimental Psychology-General, 144 , $1146-1152$

Sullivan, P. F. (2007). Spurious genetic associations. Biological Psychiatry, 61, 1121-1126.

Tang, H., Quertermous, T., Rodriguez, B., Kardia, S. L. R., Zhu, X. F., Brown, A., et al. (2005). Genetic structure, self-identified race/ethnicity, and confounding in case-control association studies. American Journal of Human Genetics, 76, 268-275.

Vul, E., \& Pashler, H. (2012). Voodoo and circularity errors. Neuroimage, 62, 945-948. 